\title{
SAMPLING IN PRACTICE: IS THE BEST RECONSTRUCTION SPACE BANDLIMITED?
}

\author{
Sathish Ramani, Dimitri Van De Ville, Michael Unser \\ Biomedical Imaging Group, \\ Ecole Polytechnique Fédérale de Lausanne (EPFL), \\ CH-1015 Lausanne, Switzerland.
}

\begin{abstract}
Shannon's sampling theory and its variants provide effective solutions to the problem of reconstructing a signal from its samples in some "shift-invariant" space, which may or may not be bandlimited. In this paper, we present some further justification for this type of representation, while addressing the issue of the specification of the best reconstruction space. We consider a realistic setting where a multidimensional signal is prefiltered prior to sampling and the samples corrupted by additive noise. We consider two formulations of the reconstruction problem. In the first deterministic approach, we determine the continuous-space function that minimizes a variational, Tikhonov-like criterion that includes a discrete data term and a suitable continuous-space regularization functional. In the second formulation, we seek the minimum mean square error (MMSE) estimation of the signal assuming that the input signal is a realization of a stationary random process. Interestingly, both approaches yield a solution included in some optimal shiftinvariant space that is generally not bandlimited. The solutions can be made equivalent by choosing a regularization operator that corresponds to the whitening filter of the process. We present some practical examples that demonstrate the optimality of the approach.
\end{abstract}

\section{INTRODUCTION}

Shannon's sampling theory provides an elegant method to perfectly reconstruct bandlimited signals from their uniform samples [1]. Since real-world signals are rarely bandlimited, the recommended procedure is to apply a lowpass filter prior to sampling to avoid aliasing. In practice, this prefilter corresponds to the impulse response of the acquisition device and is generally non-ideal. Moreover, the samples are often corrupted by measurement noise. Fortunately, it is possible to partially compensate for these non-ideal conditions using digital filtering techniques (inverse filtering or deconvolution) [3].

Mathematically, Shannon's sampling/reconstruction process in the noise-free case is equivalent to computing the orthogonal projection of the input signal $f(x)$ onto the space of bandlimited function $V=\operatorname{span}\{\operatorname{sinc}(x-k)\}_{k \in \mathbb{Z}}$ where $\operatorname{sinc}(x)$ is the impulse response of the ideal filter (to

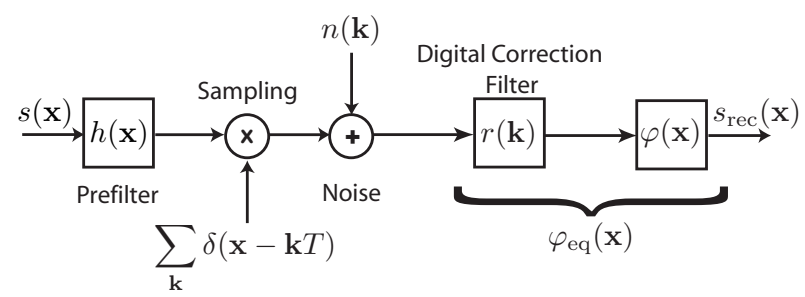

Fig. 1. Practical Sampling Setup and Reconstruction.

keep the notation simple, we are using a normalized sampling step $T=1$ ). More recently, it has been shown that one can use essentially the same ingredients (filtering and sampling) to determine the optimal approximation of the signal $f(x)$ in some more general "integer-shift invariant" space $V_{\varphi}=\operatorname{span}\{\varphi(x-k)\}_{k \in \mathbb{Z}}$ where $\varphi$ is a generating function that can be essentially arbitrary $[1,2]$. The optimal setting for the more general case is to choose an analysis function (corresponding to the impulse response of the acquisition device) that is biorthogonal to the synthesis or generating function $\varphi$. When the sampling conditions are non-ideal (e.g., the analysis and synthesis functions are not perfectly matched, and/or the samples are corrupted by noise), it is also possible to apply digital correction techniques to restore the signal. As in the classical case, this corresponds to a special kind of deconvolution, except that the determination of the restoration filter is slightly more involved because of its explicit dependence on $\varphi$ [2]. There is no major difficulty in extending these techniques to the multidimensional case, which leads to the general reconstruction method illustrated by the block diagram in Fig. 1. The bottom line is that the user is free to select the reconstruction space he wishes, provided of course that he optimizes the digital correction filter accordingly.

In this work, we consider the problem of the selection of the most appropriate reconstruction space, and whether or not it is justifiable in practice to depart from the traditional bandlimited formulation. The answer that we provide is based on the specification of two global, continuous-space optimization problems and the derivation of their optimal solution which yield the appropriate, integer-shift-invariant reconstruction framework. First, in Section 3, we adopt a 
variational point of view where we consider the minimization of a quadratic data term subject to some regularization constraint (continuous-space Tikhonov functional). This allows us to determine a reconstruction space that is globally optimum; it is tightly linked to the choice of regularization operator L, but does not depend on the regularization parameter $\lambda$ which controls the strength of the restoration filter. In Section 4, we propose an alternative stochastic formulation, which leads to the derivation of a hybrid version of the Wiener filter (i.e., discrete input, analog output), and yields the same type of solution (generally nonbandlimited). Finally, we present some experimental results in Section 5.

\section{RECONSTRUCTION SPACE}

For details on the specification of general integer-shiftinvariant reconstruction spaces, as well as for specific examples, we refer to [2]. Here, we consider the multidimensional setting with $f(\mathbf{x}) \in L_{2}\left(\mathbb{R}^{d}\right)$ (the space of finite energy functions in $d$-dimensions). The Fourier transform of $f$ is denoted by $\hat{f}(\boldsymbol{\omega})$ with $\boldsymbol{\omega} \in \mathbb{R}^{d}$. The discrete counterpart (digital signal) is the square summable sequence $c[\mathbf{k}] \in \ell_{2}\left(\mathbb{Z}^{d}\right)$ whose discrete-space Fourier transform is denoted by $C\left(e^{j \boldsymbol{\omega}}\right)$.

The reconstruction space generated by the function $\varphi(\mathbf{x})$, with $\mathbf{x} \in \mathbb{R}^{d}$, is defined as

$$
V(\varphi)=\left\{f(\mathbf{x})=\sum_{\mathbf{k} \in \mathbb{Z}^{d}} c[\mathbf{k}] \varphi(\mathbf{x}-\mathbf{k}) \mid c \in \ell_{2}\left(\mathbb{Z}^{d}\right)\right\}
$$

For the approximation to be well defined, the basis functions $\{\varphi(\mathbf{x}-\mathbf{k})\}_{\mathbf{k} \in \mathbb{Z}^{d}}$ must satisfy the two admissibility conditions:

$$
\begin{aligned}
0<C_{1} \leq & \sum_{\mathbf{k} \in \mathbb{Z}^{d}}|\hat{\varphi}(\boldsymbol{\omega}+2 \pi \mathbf{k})|^{2} \leq C_{2}<\infty \\
& \sum_{\mathbf{k} \in \mathbb{Z}^{d}}|\varphi(\mathbf{x}-\mathbf{k})|<\infty
\end{aligned}
$$

In particular, this ensures that the set of functions $\{\varphi(\mathbf{x}-\mathbf{k})\}$ forms a $L_{p}$-stable Riesz basis, for $1 \leq p \leq \infty$, and that $V(\varphi)$ is a closed subspace of $\mathrm{L}_{2}\left(\mathbb{R}^{d}\right)$. The key point is that any function $f(\mathbf{x}) \in V(\varphi)$, has a stable and unambiguous representation in terms of its coefficients $c[\mathbf{k}]$ with $\mathbf{k} \in \mathbb{Z}^{d}$.

Given such a space, the least squares (LS) approximation $\mathrm{P}_{V}(f)$ of $f$ exists and is equal to the orthogonal projection of $f$ onto $V(\varphi)$, given by

$$
\mathrm{P}_{V}(f)=\sum_{\mathbf{k} \in \mathbb{Z}^{d}}\langle\tilde{\varphi}(\cdot-\mathbf{k}), f\rangle \varphi(\mathbf{x}-\mathbf{k})
$$

where $\tilde{\varphi}(\mathbf{x}) \in V(\varphi)$ is the dual of $\varphi(\mathbf{x})$.
It is important to note that the generator $\varphi$ of $V(\varphi)$ is by no means unique. In fact, it can be shown that any function of the form

$$
\varphi_{\mathrm{eq}}(\mathbf{x})=\sum_{\mathbf{k} \in \mathbb{Z}^{d}} q[\mathbf{k}] \varphi(\mathbf{x}-\mathbf{k}),
$$

will generate an equivalent basis of $V(\varphi)$, if and only if

$$
0<A \leq\left|Q\left(e^{j \omega}\right)\right| \leq B<\infty,
$$

where $Q\left(e^{j \boldsymbol{\omega}}\right)$ is the Fourier transform of $q[\mathbf{k}]$.

\section{TIKHONOV-REGULARIZED RECONSTRUCTION}

The measurement model associated with Fig. 1 is

$$
g[\mathbf{k}]=(h * s)(\mathbf{k})+n[\mathbf{k}],
$$

where $g[\mathbf{k}]$ are the measurements, and $(h * s)(\mathbf{k})$ are the samples of prefiltered signal; $n[\mathbf{k}]$ is an discrete additive, zero-mean noise component with variance $\sigma^{2}$.

Given the noisy prefiltered samples $g[\mathbf{k}]$, our goal is to recover the unknown deterministic signal, $s(\mathbf{x})$, which is defined in the continuous domain. To this end, we will make use of the a priori information that the energy functional, $\|\mathrm{L} s\|_{L_{2}}^{2}=\int_{\mathbf{x} \in \mathbb{R}^{d}}|\mathrm{~L}\{s(\mathbf{x})\}|^{2} d \mathbf{x}$ where $\mathrm{L}$ is an appropriate differential operator, is bounded by some constant $C_{0}$. The signal is then determined by fitting the model to the data. This leads to the minimization of the following cost functional

$$
\min _{u(\mathbf{x}) \in L_{2}} \sum_{\mathbf{k} \in \mathbb{Z}^{d}}|g[\mathbf{k}]-(h * u)(\mathbf{k})|^{2}+\lambda\|\mathrm{L} u\|_{\mathrm{L}_{2}}^{2},
$$

where $\lambda$ is the Lagrange multiplier. The above criterion ensures that the resulting continuous-space estimate is consistent in the least squares sense (first data term), and also regular (second term) in the sense of its smoothest energy (Tikhonov functional), $\|\mathrm{L} u\|_{L_{2}}^{2}$, being reasonably small, depending on the magnitude of $\lambda=\lambda\left(C_{0}\right)$. We will therefore refer to $\mathrm{L}$ as the regularization operator, and to $\lambda$ as the regularization parameter.

Our first result is that the general solution of this problem is included in some "optimal" shift-invariant space $V(\phi)$ and that the coefficients of the representation can be obtained by digital filtering.

Theorem 1 Let $\phi$ be an admissible generating function that satisfies the optimality condition

$$
\mathrm{L}^{*} \mathrm{~L}\{\phi(\mathbf{x})\}=\sum_{\mathbf{k} \in \mathbb{Z}^{d}} p[\mathbf{k}] \bar{h}(\mathbf{x}-\mathbf{k})
$$

for some $p[\mathbf{k}] \in \ell_{1}\left(\mathbb{Z}^{d}\right)$ with the notation $\bar{h}(\mathbf{x})=h(-\mathbf{x})$. Then, the solution of the Tikhonov problem (8) can be determined as

$$
s_{\mathrm{rec}}(\mathbf{x})=\sum_{\mathbf{k} \in \mathbb{Z}^{d}}\left(r_{\lambda} * g\right)[\mathbf{k}] \phi(\mathbf{x}-\mathbf{k}) .
$$


where the frequency response of the optimal restoration filter $r_{\lambda}[\mathbf{k}]$ is

$$
R_{\lambda}\left(e^{j \boldsymbol{\omega}}\right)=\frac{1}{\sum_{\mathbf{k} \in \mathbb{Z}^{d}} \hat{\phi}(\boldsymbol{\omega}+2 \pi \mathbf{k}) \hat{h}(\boldsymbol{\omega}+2 \pi \mathbf{k})+\lambda P\left(e^{j \boldsymbol{\omega}}\right)},
$$

with $P\left(e^{j \omega}\right)$, the Fourier transform of $p[\mathbf{k}]$. The solution is well-defined and unique provided that the denominator of (11) is non-vanishing.

Proof: Because of lack of space, we are only providing the proof of the second part of the theorem, namely, the derivation of the optimal solution in the space $V(\phi)$, where $\phi$ satisfies (9). The fact that this solution is also globally optimum - that is, the best among all possible functionsis a more sophisticated result whose proof will be published in a forthcoming paper. Here, we simply assume that $u \in$ $V(\phi)$, which allows us to write the cost function in terms of the following $\ell_{2}$-inner products,

$$
\begin{aligned}
\epsilon= & \|g-(h * u)\|_{\ell_{2}}^{2}+\lambda\|\mathrm{L} u\|_{\mathrm{L}_{2}}^{2} \\
= & \langle g, g\rangle_{\ell_{2}}-2\left\langle\bar{b}_{h} * g, c\right\rangle_{\ell_{2}}+\left\langle\bar{b}_{h} * b_{h} * c, c\right\rangle_{\ell_{2}} \\
& +\lambda\left\langle\bar{b}_{h} * p * c, c\right\rangle_{\ell_{2}},
\end{aligned}
$$

where $b_{h}[\mathbf{k}]=(h * \phi)(\mathbf{k})$.

The optimal coefficients are obtained by setting the partial derivative of $\epsilon$ with respect to $c[\mathbf{k}]$ to zero, which yields,

$$
\left(\bar{b}_{h} * b_{h} * c\right)[\mathbf{k}]+\lambda\left(\bar{b}_{h} * p * c\right)[\mathbf{k}]=\left(\bar{b}_{h} * g\right)[\mathbf{k}] .
$$

Rewriting the above equality in the Fourier domain, we obtain the desired result.

This result is conceptually pleasing because the continuous-space optimization problem (8) does not make any a priori assumption on the form of the reconstruction. The shift-invariant structure of the solution comes out as a result of the mathematical optimization. The optimal reconstruction space is specified by the optimality condition (9). Note that the fundamental solution (i.e., $p[\mathbf{k}]=\delta[\mathbf{k}]$ ) is $\phi(\mathbf{x})=(\bar{h} * \rho)(\mathbf{x})$ where $\rho$ is the Green function of the selfadjoint operator $\mathrm{L}^{*} \mathrm{~L}$, and that this generator is generally not bandlimited.

If we write the solution (10) in the Fourier domain, we obtain

$$
\hat{s}_{\mathrm{rec}}(\boldsymbol{\omega})=\hat{\phi}_{\mathrm{eq}, \lambda}(\boldsymbol{\omega}) \cdot G\left(e^{j \boldsymbol{\omega}}\right)
$$

where

$$
\hat{\phi}_{\mathrm{eq}, \lambda}(\boldsymbol{\omega})=\frac{\hat{\phi}(\boldsymbol{\omega})}{\sum_{\mathbf{k} \in \mathbb{Z}^{d}} \hat{\phi}(\boldsymbol{\omega}+2 \pi \mathbf{k}) \hat{h}(\boldsymbol{\omega}+2 \pi \mathbf{k})+\lambda P\left(e^{j \boldsymbol{\omega}}\right)},
$$

is the equivalent basis function that needs to be applied to the measurements $G\left(e^{j \omega}\right)$ to produce the continuous-space signal reconstruction in Fig. 1.

\section{STATISTICAL FORMULATION}

We now propose another complementary stochastic formulation of the reconstruction problem that essentially provides the same type of solution. The measurement equation (7) is the same as before, but the signal $s(\mathbf{x})$ is now assumed to be a realization of a stationary process with autocorrelation function $c_{s}(\mathbf{x})=E\left\{s\left(\mathbf{x}^{\prime}\right) s\left(\mathbf{x}^{\prime}+\mathbf{x}\right)\right\}$. The measurements are corrupted by discrete Gaussian stationary noise. We also assume that the signal and noise are uncorrelated. The optimal (MMSE) solution of the estimation problem is given by a hybrid version of the Wiener filter where the input, $g[\mathbf{k}]$, is discrete and the output, $s_{\text {rec }}(\mathbf{x})$, analog.

Theorem 2 Let us consider the measurement model (7) where $s(\mathbf{x})$ (continuous-space signal) and $n[\mathbf{k}]$ (discrete measurement noise) are realization of stationary processes with spectral power density $\hat{c}_{s}(\boldsymbol{\omega})$ and $C_{n}\left(e^{j \boldsymbol{\omega}}\right)$, respectively. Then, the linear MMSE estimate of $s$ at any location $\mathbf{x}=\mathbf{x}_{0}$, given the measurements $\{g[\mathbf{k}]\}_{\mathbf{k} \in \mathbb{Z}^{d}}$, is included in the optimal shift-invariant space generated by

$$
\phi(\mathbf{x})=\left(\bar{h} * c_{s}\right)(\mathbf{x}),
$$

where $\bar{h}(\mathbf{x})=h(-\mathbf{x})$. The Fourier domain reconstruction formula is the same as (14) with

$$
\hat{\phi}_{\mathrm{eq}}(\boldsymbol{\omega})=\frac{\hat{\phi}(\boldsymbol{\omega})}{\sum_{\mathbf{k} \in \mathbb{Z}^{d}} \hat{\phi}(\boldsymbol{\omega}+2 \pi \mathbf{k}) \hat{h}(\boldsymbol{\omega}+2 \pi \mathbf{k})+C_{n}\left(e^{j \boldsymbol{\omega}}\right)} .
$$

Proof: Optimality is achieved by minimizing the error

$$
\epsilon_{s}=E\left\{\left|s\left(\mathbf{x}_{0}\right)-\sum_{\mathbf{k} \in \mathbb{Z}^{d}} g[\mathbf{k}] w\left(\mathbf{x}_{0}-\mathbf{k}\right)\right|^{2}\right\}
$$

where $w(\mathbf{x})$ is the continuous-space reconstruction filter. The spectral power density of $g[\mathbf{k}]$ is

$$
C_{g}\left(e^{j \boldsymbol{\omega}}\right)=\sum_{\mathbf{k} \in \mathbb{Z}^{d}} \hat{c}_{s}(\boldsymbol{\omega}+2 \pi \mathbf{k})|\hat{h}(\boldsymbol{\omega}+2 \pi \mathbf{k})|^{2}+C_{n}\left(e^{j \boldsymbol{\omega}}\right) .
$$

Using the Wiener-Khintchine theorem, the mean square error (18) can be expressed in the Fourier domain as,

$$
\begin{aligned}
\epsilon_{s}= & \int_{\boldsymbol{\omega} \in \mathbb{R}^{d}}|\hat{w}(\boldsymbol{\omega})|^{2} C_{g}\left(e^{j \boldsymbol{\omega}}\right) \mathbf{d} \boldsymbol{\omega} \\
& -2 \operatorname{Re}\left\{\int_{\boldsymbol{\omega} \in \mathbb{R}^{d}} \hat{w}^{*}(\boldsymbol{\omega}) \hat{h}^{*}(\boldsymbol{\omega}) \hat{c}_{s}(\boldsymbol{\omega}) \mathbf{d} \boldsymbol{\omega}\right\} \\
& +\int_{\boldsymbol{\omega} \in \mathbb{R}^{d}} \hat{c}_{s}(\boldsymbol{\omega}) \mathbf{d} \boldsymbol{\omega} .
\end{aligned}
$$


Assuming that $C_{g}\left(e^{j \omega}\right)$ is non vanishing (a sufficient condition is $C_{n}\left(e^{j \boldsymbol{\omega}}\right)>0$ ), we rewrite (19) as,

$$
\begin{aligned}
\epsilon_{s} & =\int_{\boldsymbol{\omega} \in \mathbb{R}^{d}} C_{g}\left(e^{j \boldsymbol{\omega}}\right)\left|\hat{w}(\boldsymbol{\omega})-\frac{\hat{h}^{*}(\boldsymbol{\omega}) \hat{c}_{s}(\boldsymbol{\omega})}{C_{g}\left(e^{j \boldsymbol{\omega}}\right)}\right|^{2} \mathbf{d} \boldsymbol{\omega} \\
& +\int_{\boldsymbol{\omega} \in \mathbb{R}^{d}}\left(\hat{c}_{s}(\boldsymbol{\omega})-\frac{\left|\hat{h}^{*}(\boldsymbol{\omega}) \hat{c}_{s}(\boldsymbol{\omega})\right|^{2}}{C_{g}\left(e^{j \boldsymbol{\omega}}\right)}\right) \mathbf{d} \boldsymbol{\omega} .
\end{aligned}
$$

Since $C_{g}\left(e^{j \omega}\right)>0, \forall \boldsymbol{\omega} \in \mathbb{R}^{d}$ and the second term in (20) is fixed, $\epsilon_{s}$ is minimum when the first term vanishes, that is, iff

$$
\hat{w}(\boldsymbol{\omega})=\frac{\hat{h}^{*}(\boldsymbol{\omega}) \hat{c}_{s}(\boldsymbol{\omega})}{\sum_{\mathbf{k} \in \mathbb{Z}^{d}} \hat{c}_{s}(\boldsymbol{\omega}+2 \pi \mathbf{k})|\hat{h}(\boldsymbol{\omega}+2 \pi \mathbf{k})|^{2}+C_{n}\left(e^{j \boldsymbol{\omega}}\right)},
$$

which is equivalent to (17) with $\phi$ given by (16).

Note that the structure of the solution is very much the same as in Theorem 1. Here too, the optimal reconstruction space will generally be non-bandlimited unless either $h$ or $s$ are bandlimited to start with.

By comparing the deterministic and stochastic solutions in Theorem 1 and 2, we can obtain the following equivalence, which corresponds to the case $P\left(e^{j \omega}\right)=1$ and $C_{n}\left(e^{j \boldsymbol{\omega}}\right)=c_{0} \cdot \sigma^{2}$.

Corollary 1 The Tikhonov and Wiener solutions are functionally equivalent provided that

(1) the operator $\mathrm{L}$ is the whitening filter of the continuous-space stochastic process, $s(\mathbf{x})$, i.e., $\mathrm{L}^{*} \mathrm{~L}\left\{c_{s}(\mathbf{x})\right\}=\sigma_{0}^{2} \delta(\mathbf{x})$, and

(2) the measurement noise is white, with variance $\sigma^{2}$.

The corresponding optimal regularization parameter is $\lambda_{\mathrm{opt}}=\frac{\sigma^{2}}{\sigma_{0}^{2}}$.

This last result is interesting because it helps us select the best regularization operator $\mathrm{L}$ and parameter $\lambda$ for the Tikhonov solution in Theorem 1.

\section{RESULTS}

For evaluation purposes, we chose a phase contrast micrograph of a biological specimen. Its autocorrelation function estimated at the initial resolution was assumed to be known. A Gaussian smoothing prefilter with $\sigma_{h}=0.25$ was used and the image was downsampled by a factor of 2. Zero mean white-Gaussian noise of suitable variance was added to the downsampled image so as to achieve the desired SNR. The original image was then reconstructed from the noisy samples using the three reconstruction methods, (a) optimal non-bandlimited Wiener (proposed in this work), (b) textbook Wiener with bandlimited spectra, and (c) Classical Shannon's bandlimited reconstruction (plain sinc-interpolation), respectively.

The SNR of the reconstructed image was computed for varying SNR of the noisy sampled image. Fig. 2 shows the

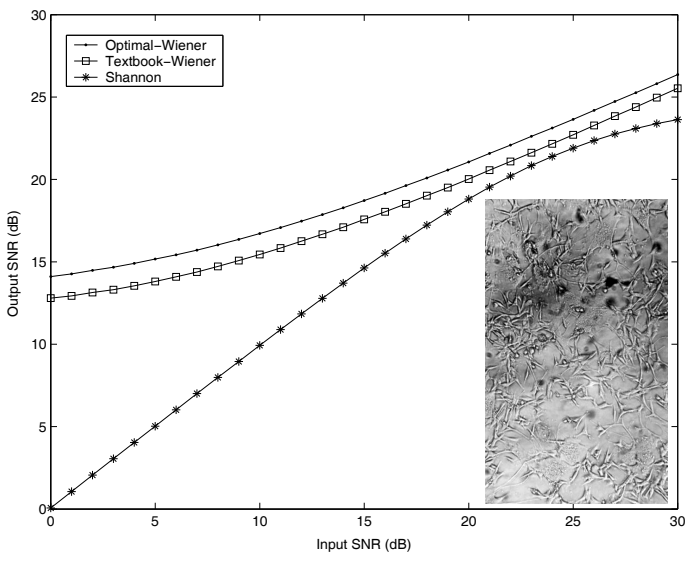

Fig. 2. Performance of reconstruction methods.

variation of the SNR of the reconstructed image for each method. The inset shows the image used for our experiment. Since our image was not bandlimited, the methods (b) and especially (c), perform less well than the proposed method. The optimal non-bandlimited Wiener reconstruction clearly outperforms the other two methods confirming our theoretical observations.

\section{CONCLUSIONS}

We have presented new theoretical arguments that further justify the use of shift-invariant representations of signals. We have specified the "best" reconstruction space for the deterministic (Tikhonov) and stochastic (Wiener) formulations. We have shown that, in both cases, the expansion coefficients of the signal could be determined effectively by digital filtering. We also established an equivalence between the reconstruction methods for stochastic and deterministic signals by showing that the optimal basis functions are equivalent generators of the same function space.

Our experimental results confirm that the optimal reconstruction procedure suggested in this work can be superior to the classical reconstruction procedure for bandlimited signals.

\section{ACKNOWLEDGMENTS}

This work is funded in part by the grant 20020-101821 from the Swiss National Science Foundation (SNSF).

\section{REFERENCES}

[1] M. Unser, "Sampling-50 Years After Shannon," Proceedings of IEEE, vol. 88, no. 4, pp. 569-587, 2000.

[2] M. Unser, A. Aldroubi, "A General Sampling Theory for Nonideal Acquisition Devices," IEEE Transactions on Signal Processing, vol. 42, no. 11, pp. 2915-2925, 1994.

[3] M. R. Banham and A. K. Katsaggelos, "Digital Image Restoration," IEEE Signal Processing Magazine, vol. 14, no. 2, pp. 24-41, 1997. 